\title{
The validity of the kinetic collection equation revisited - Part 3: Sol-gel transition under turbulent conditions
}

\author{
L. Alfonso ${ }^{1}$, G. B. Raga ${ }^{2}$, and D. Baumgardner ${ }^{2}$ \\ ${ }^{1}$ Universidad Autónoma de la Ciudad de México, México City, 09790, Mexico \\ ${ }^{2}$ Centro de Ciencias de la Atmósfera, Universidad Nacional Autónoma de México, México City, 04510, Mexico
}

Correspondence to: L. Alfonso (lesterson@yahoo.com)

Received: 29 September 2011 - Published in Atmos. Chem. Phys. Discuss.: 23 January 2012

Revised: 20 November 2012 - Accepted: 6 December 2012 - Published: 16 January 2013

\begin{abstract}
Warm rain in real clouds is produced by the collision and coalescence of an initial population of small droplets. The production of rain in warm cumulus clouds is still one of the open problems in cloud physics, and although several mechanisms have been proposed in the past, at present there is no complete explanation for the rapid growth of cloud droplets within the size range of diameters from 10 to $50 \mu \mathrm{m}$. By using a collection kernel enhanced by turbulence and a fully stochastic simulation method, the formation of a runaway droplet is modeled through the turbulent collection process. When the runaway droplet forms, the traditional calculation using the kinetic collection equation is no longer valid, since the assumption of a continuous distribution breaks down. There is in essence a phase transition in the system from a continuous distribution to a continuous distribution plus a runaway droplet. This transition can be associated to gelation (also called sol-gel transition) and is proposed here as a mechanism for the formation of large droplets required to trigger warm rain development in cumulus clouds. The fully stochastic turbulent model reveals gelation and the formation of a droplet with mass comparable to the mass of the initial system. The time when the sol-gel transition occurs is estimated with a Monte Carlo method when the parameter $\rho$ (the ratio of the standard deviation for the largest droplet mass over all the realizations to the averaged value) reaches its maximum value. Moreover, we show that the non-turbulent case does not exhibit the sol-gel transition that can account for the impossibility of producing raindrop embryos in such a system. In the context of cloud physics theory, gelation can be interpreted as the formation of the "lucky droplet" that grows at a much faster rate than the rest of the population and becomes the embryo for runaway raindrops.
\end{abstract}

\section{Introduction}

Whether the formation of large droplets trigger the production of rain in warm cumulus clouds remains one of the open problems in cloud physics. Although several mechanisms have been proposed (Pruppacher and Klett, 1997), at present there is no complete explanation for the rapid growth of cloud droplets within the size range of diameters from 10 to $50 \mu \mathrm{m}$.

Some existing hypotheses try to explain the formation of these large droplets by condensation of water vapor molecules onto droplet embryos (Khain et al., 2000). Many other studies include droplet coalescence as an important factor, mainly through two mechanisms: (i) the collision of large droplets growing on giant and ultra-giant nuclei, and (ii) the self-broadening of the droplet spectra by collisions between cloud droplets. Regarding this second mechanism, it has been emphasized by experimental (Vohl et al., 1999) and theoretical (Pinsky et al., 1999, 2000) studies that there is a significant acceleration of droplet growth rate by collisions in a turbulent flow, with collision efficiencies that may reach values 10 times larger than in the pure gravity case.

In this study we focus on a model for the growth of cloud droplets by a fully stochastic turbulent collision-coalescence process and we will show that this model reveals a solgel transition in the system and the formation of runaway droplets. The term sol-gel transition (also known as gelation) is not very familiar in the context of cloud physics, and can be defined as a change from a system with a continuous droplet distribution to one with a continuous distribution plus a massive (runaway) droplet. 


\subsection{Approach of previous studies}

The kinetic collection or coagulation equation (hereafter $\mathrm{KCE}$ ) has long been used to model the time evolution of droplet size distributions due to collection events. The discrete version of this equation can be written as (Pruppacher and Klett, 1997)

$$
\begin{gathered}
\frac{\partial N(i, t)}{\partial t}=\frac{1}{2} \sum_{j=1}^{i-1} K(i-j, j) N(i-j) N(j) \\
-N(i) \sum_{j=1}^{\infty} K(i, j) N(j)
\end{gathered}
$$

where $N(i, t)$ is the average number of droplets with mass $x_{i}$, and $K(i, j)$ is the coagulation kernel related to the probability of coalescence of two drops of masses $x_{i}$ and $x_{j}$. In Eq. (1), the time rate of change of the average number of droplets with mass $x_{i}$ is determined as the difference between two terms: the first term describes the average rate of production of droplets of mass $x_{i}$ due to coalescence between pairs of droplets whose masses add up to mass $x_{i}$, and the second term describes the average rate of depletion of droplets with mass $x_{i}$ due to their collisions and coalescence with other droplets. Although the term stochastic has been associated with the KCE for historical reasons, it is clearly deterministic and has no stochastic correlations or fluctuations included.

As a matter of fact, the average spectrum obtained from Eq. (1) and the ensemble averaged spectrum obtained over different realizations of the stochastic collection process are different. The solution to the KCE and the expected values calculated from the stochastic process are only equal if the covariances are omitted from the probabilistic model, as shown in Bayewitz et al. (1974) and Tanaka and Nakazawa (1993). It is only when this condition is fulfilled that the deterministic solution provided by Eq. (1) corresponds to the average value of $n_{k}$ over many realizations of the stochastic process.

The relevance of fluctuations compared to mean particle growth was discussed many years ago by Telford (1955), Robertson (1974) and Young (1975) and more recently by Kostinski and Shaw (2005). Telford (1955) introduced the probabilistic interpretation assuming that the concentration of droplets available for collection remains unchanged, and that the collecting droplets do not interact among themselves. Robertson (1974) basically follows the same approach as in Telford (1955), using a Monte Carlo procedure to calculate the collection process, also considered an idealized cloud of constant volume and assumed that only drop-droplet interactions are permitted. However, the method for selecting the time between coalescence events is not completely stochastic. Kostinski and Shaw (2005) adopted a version of Telford's approach to illustrate the influence of stochastic fluctuations, which can lead to a factor-of-10 acceleration in the growth of a few lucky drops. However, all these studies follow a quasistochastic approach (see the complete analysis in Gillespie, 1975a), which ignores fluctuations in the droplet mass spectrum.

The full stochastic approach was used by Bayewitz et al. (1974) and Gillespie (1975b), using a constant collection kernel. The stochastic completeness of the KCE was revisited more recently by Wang et al. (2006) for realistic collection kernels, resulting in a novel deduction of a master equation. They demonstrated that, for a system of finite liquid mass and narrow initial size distribution, both stochastic correlations and fluctuations are important.

\subsection{Approach of this study}

We apply the pure stochastic model (Gillespie, 1975a) to perform a fully stochastic collision-coalescence calculation (see Appendix A) following Laurenzi et al. (2002), that inherently incorporates all stochastic correlations present in the collection process. This computational procedure is rigorously based on the probability distribution instead of using the kinetic collection equation.

As the collection process is stochastic in nature, it is, therefore, more accurately described by the master equation for the joint probability distribution $P\left(n_{1}, n_{2}, \ldots, n_{k}, \ldots, t\right)$ for the occupation numbers $\bar{n}=\left(n_{1}, n_{2}, \ldots, n_{k}, \ldots\right)$ at time $t$. This equation can be written as (Bayewitz et al., 1974)

$$
\begin{aligned}
& \frac{\partial P(\bar{n})}{\partial t}=\sum_{i=1}^{N} \sum_{j=i+1}^{N} K(i, j)\left(n_{i}+1\right)\left(n_{j}+1\right) \\
& P\left(\ldots, n_{i}+1, \ldots, n_{j}+1, \ldots, n_{i+j}-1, \ldots ; t\right) \\
& +\sum_{i=1}^{N} \frac{1}{2} K(i, i)\left(n_{i}+2\right)\left(n_{i}+1\right) P\left(\ldots, n_{i}+2, \ldots, n_{2 i}-1, \ldots ; t\right) \\
& -\sum_{i=1}^{N} \sum_{j=i+1}^{N} K(i, j) n_{i} n_{j} P(\bar{n} ; t) \\
& -\sum_{i=1}^{N} \frac{1}{2} K(i, i) n_{i}\left(n_{i}-1\right) P(\bar{n} ; t)
\end{aligned}
$$

Note that the KCE can be recovered from Eq. (2) by considering the mean value of $n_{k}$ :

$\left\langle n_{k}\right\rangle=\sum_{\bar{n}} n_{k} P(\bar{n} ; t)$,

and assuming, as in Bayewitz et al. (1974), that $\left\langle n_{i} n_{j}\right\rangle=$ $\left\langle n_{i}\right\rangle\left\langle n_{j}\right\rangle$.

The first moment of the distribution of $N(i, t)$ corresponds to the total mass in the system $\left(M_{1}\right)$; the second moment $\left(M_{2}\right)$ for a number of droplet categories or sizes of the discrete distribution $\left(N_{\mathrm{d}}\right)$, is defined as 
$M_{2}(t)=\sum_{i=1}^{N_{\mathrm{d}}} x_{i}^{2} N(i, t)$.

Note that $M_{2}$ may become undefined if the initial number of droplets is small or if the kernel $K(i, j)$ increases sufficiently rapidly with $x_{i}$ and $x_{j}$. In that case, the total mass in the system $\left(M_{1}\right)$ starts to decrease. This is usually interpreted to mean that a macroscopic runaway droplet has formed (known as a $\mathrm{gel}$ ) and the system exhibits a phase solgel transition (also called gelation). After this point in time, the average calculated from the stochastic process will differ from the average obtained from the KCE (Eq. 1), and there is a transition from a system with a continuous droplet distribution to one with a continuous distribution plus a massive runaway droplet.

Note that when gelation occurs, mass conservation is expected to break down. The gelation time, $T_{\text {gel }}$, is defined as as the largest time such that the discrete model (1) has a solution with $M_{1}(t) \equiv M_{1}(0)$ for $t<T_{\text {gel }}$ and $M_{1}(t)<M_{1}(0)$ for $t>T_{\mathrm{gel}}$, where $M_{1}$ is the total mass of the system.

Since analytical expressions for the gelation time only exist for very simple kernels, Inaba et al. (1999) proposed that it could be estimated numerically by Monte Carlo simulations (an approach followed in Alfonso et al., 2008, 2010).

The gelation time, $T_{\text {gel }}$, is the point in time when the maximum of the ratio $\rho$ (see Eq. 5) is reached:

$\rho=\sigma\left(M_{\mathrm{L} 1}\right) / M_{\mathrm{L} 1}$,

where $M_{\mathrm{L} 1}$ is the ensemble mean of the mass of the largest droplet and $\sigma\left(M_{\mathrm{L} 1}\right)$ is the standard deviation for the largest droplet mass $(\sigma)$ over all the realizations. This standard deviation is calculated as

$\sigma\left(M_{\mathrm{L} 1}\right)=\sqrt{\frac{1}{K} \sum_{i=1}^{K}\left(M_{\mathrm{L} 1}^{i}-M_{\mathrm{L} 1}\right)^{2}}$

In Eq. (6), $M_{\mathrm{L} 1}^{i}$ is the largest droplet mass for each realization and $K$ is the number of realizations of the Monte Carlo algorithm.

The gelation time, estimated as described above, can be interpreted as the expected time of formation of the "lucky droplet" that becomes the embryo for runaway raindrops, and in this study, it is estimated from Monte Carlo simulations under turbulent conditions.

\section{Results}

It is well known that the KCE (Eq. 1) only has analytical solutions for a few selected kernels such as the product kernel $K(i, j)=C x_{i} x_{j}$. Note that the validity of the KCE will break down once gelation occurs, since the underlying assumption of a continuous distribution is no longer true. We demonstrate that the time when the parameter $\rho$ (Eq. 5) reaches its maximum value is a good estimate of the gelation time $T_{\mathrm{gel}}$. We first present our results using the fully stochastic model for a mono-dispersed initial droplet distribution (Sect. 2.1), then compare the results from the fully stochastic simulation with the results from the KCE with turbulence for a bidispersed distribution, and (Sect. 2.2) and (Sect. 2.3) present the results for the non-turbulent case. Finally, in Sect. 2.4 we calculate the time evolution of sol concentration, evaluate the performance of the stochastic algorithm and calculate the Monte Carlo errors.

\subsection{Results for the product collection kernel using a fully stochastic model}

The calculations were performed for an initial mono-disperse distribution of 100 droplets of $14 \mu \mathrm{m}$ in radius (droplet mass $1.15 \times 10^{-8} \mathrm{~g}$ ), with $C=5.49 \times 10^{10} \mathrm{~cm}^{3} \mathrm{~g}^{-2} \mathrm{~s}^{-1}$ (Alfonso et al., 2008) in a volume of one cubic centimeter. This initial concentration is typical of maritime cumulus clouds and corresponds to a liquid water content (LWC) of about $1.15 \mathrm{~g} \mathrm{~m}^{-3}$. The time evolution of the parameter $\rho$ was estimated from 1000 realizations $(K=1000)$ of the Gillespie (1976) Monte Carlo algorithm. The results of this simulation are displayed in Fig. 1 and we observe that the maximum of $\rho$ (solid line) was obtained at $\tau=1335 \mathrm{~s}$. Independently, the gelation time can be obtained analytically (Drake and Wright, 1972) from

$$
\begin{aligned}
& M_{2}(\tau)=\frac{M_{2}(0)}{1-C \times M_{2}(0) \tau} \\
& T_{\text {gel }}=\left[C \times M_{2}\left(t_{0}\right)\right]^{-1}
\end{aligned}
$$

and was found to be $1379 \mathrm{~s}$, very close (less than $5 \%$ difference) to the time when $\rho$ reaches its maximum value. After this time, the largest droplet continues to grow by accretion of smaller droplets and the total mass $M_{1}$ predicted by the KCE starts to decrease (Wetherill, 1990), as seen in Fig. 1. Thus, the numerical method provides a reliable approximation of the gelation time.

\subsection{Comparison with KCE results under turbulent conditions}

In many models, it is usual to model collisions between droplets under idealized, pure gravity conditions with a collection kernel of the form

$K_{\mathrm{g}}\left(x_{i}, x_{j}\right)=\pi\left(r_{i}+r_{j}\right)^{2}\left|V\left(x_{i}\right)-V\left(x_{j}\right)\right| E\left(r_{i}, r_{j}\right)$.

This hydrodynamic kernel under pure gravity conditions $\left(K_{\mathrm{g}}\right)$ does not take into account the turbulence effects, and considers that droplets with different masses $\left(x_{i}\right.$ and $x_{j}$, and 


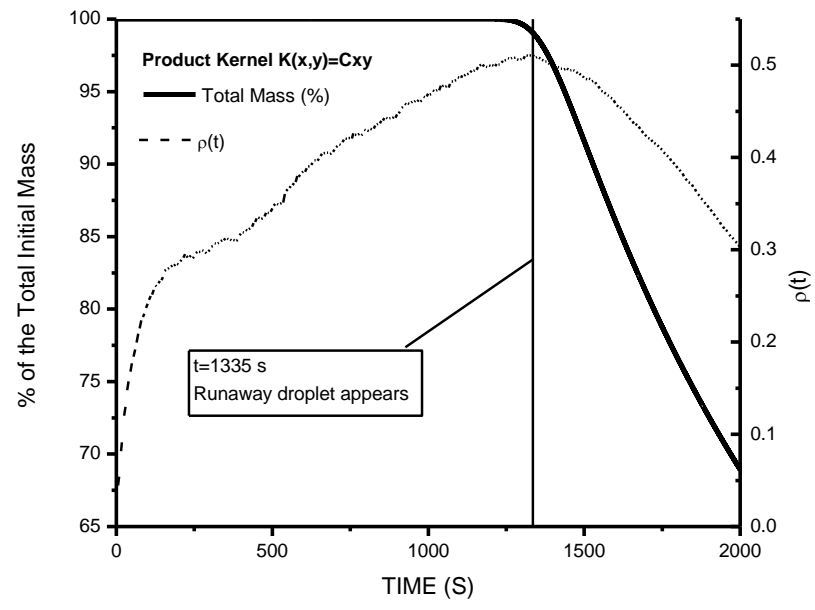

Fig. 1. Time evolution of the parameter $\rho$ defined in Eq. (5) (dashed line and right axis) and the total mass (solid line and left axis) calculated from the numerical solution of the KCE. The results were obtained for the product kernel $K(x, y)=C x y,(C=$ $\left.5.49 \times 10^{10} \mathrm{~cm}^{3} \mathrm{~g}^{-2} \mathrm{~s}^{-1}\right)$.

corresponding radii $r_{i}$ and $r_{j}$ ) have different settling velocities.

In turbulent air, the hydrodynamic kernel $K_{\mathrm{g}}$ (Eq. 9) can be enhanced due to (a) an increase in relative velocity between droplets (transport effect) and (b) an increase in the collision efficiency (the droplet hydrodynamic interaction effect). These effects were considered by implementing the turbulence-induced collision enhancement factor $P_{\text {Turb }}\left(x_{i}\right.$, $x_{j}$ ) as calculated in Pinsky et al. (2008) for a cumulonimbus, with dissipation rate $\varepsilon=0.1 \mathrm{~m}^{2} \mathrm{~s}^{-3}$ and Reynolds number $R e_{\lambda}=2 \times 10^{4}$, and for cloud droplets with radii $\leq 21 \mu \mathrm{m}$. Consequently, the turbulent collection kernel has the form

$K_{\text {Turb }}\left(x_{i}, x_{j}\right)=P_{\text {Turb }}\left(x_{i}, x_{j}\right) K_{\mathrm{g}}\left(x_{i}, x_{j}\right)$.

The simulation of collisions in a turbulent cloud was performed considering a cloud volume of $1 \mathrm{~cm}^{3}$ and an initial bi-dispersed droplet distribution: 150 droplets of $10 \mu \mathrm{m}$ in radius, and another 150 droplets of $12.6 \mu \mathrm{m}$ in radius, corresponding to a LWC of $1.9 \mathrm{~g} \mathrm{~m}^{-3}$. Collision efficiencies $E\left(r_{i}\right.$, $r_{j}$ ) in Eq. (9) are calculated according to Hall (1980).

The evolution of the total mass obtained by solving the $\mathrm{KCE}$ (Eq. 1) numerically under these conditions is shown in Fig. 2 (solid line). Note that the total mass (expressed in \% of the initial total mass) is no longer conserved after $1000 \mathrm{~s}$. The behavior of the parameter $\rho$ (Eq. 5) evaluated from 1000 realizations of the Monte Carlo algorithm is also shown in Fig. 2 (dashed line), indicating a maximum at $1055 \mathrm{~s}$, again very close (about $5.5 \%$ difference) to the time when the numerical solution of Eq. (1) breaks down. These results clearly indicate that the sol-gel transition and the formation of a runaway droplet took place around $1000 \mathrm{~s}$ and that the parameter $\rho$ (Eq. 5) can be used as an estimator of the gelation time when realistic turbulence collection kernels are used.

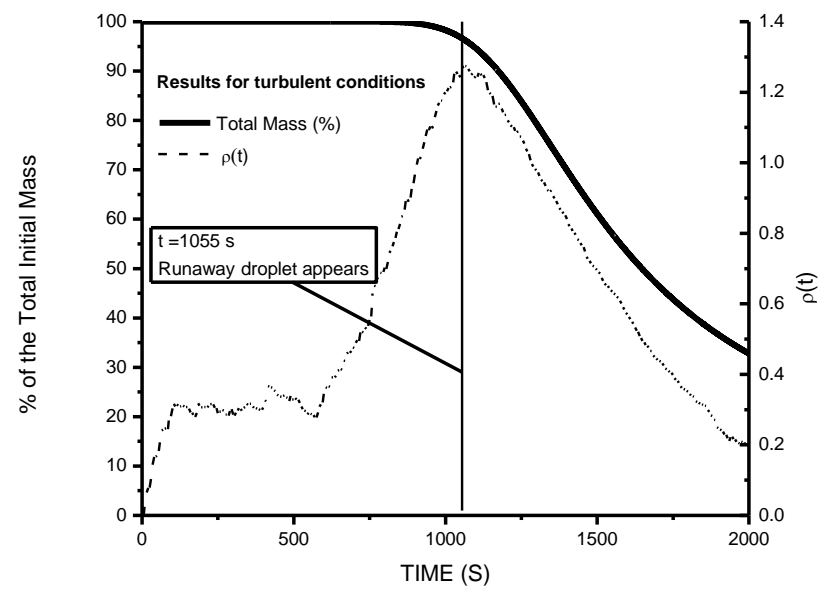

Fig. 2. Time evolution of total mass calculated from the numerical solution of the kinetic collection equation (KCE) for turbulent collision-coalescence (solid line and left axis) and the parameter $\rho$ (dashed line and right axis) estimated from the Monte Carlo algorithm.

\subsection{Comparison with $\mathrm{KCE}$ results under non-turbulent, pure gravity conditions}

To emphasize the importance of the turbulence enhancement in the collection process, an additional simulation was performed for non-turbulent flow under the Earth's gravitational field with the same initial conditions as in Sect. 2.2. The total mass from the numerical integration of the KCE after $2000 \mathrm{~s}$ was found to be equal to $99.88 \%$ of the initial mass (see Fig. 3), illustrating mass conservation for this case. Furthermore, the parameter $\rho$ (Eq. 5) estimated from the stochastic model never reaches its maximum, confirming that the sol-gel transition does not take place under these conditions. Therefore, the results suggest that the collision-coalescence process under non-turbulent conditions does not show a solgel phase transition for time intervals relevant to the problem under discussion (warm rain initiation), with no generation of a runaway droplet.

\subsection{Time evolution of sol concentration and performance of the Monte Carlo algorithm}

In order to check the performance of the Monte Carlo algorithm for the turbulent collection kernel (Eq. 10), the ensemble averages (at each time) for the sol concentration over 1000 realizations were compared with the numerical solution of the KCE. As can be observed in Fig. 4, the time evolution of the sol concentration predicted by the two models was almost identical until the sol-gel transition approaches and the breakdown of the KCE takes place.

Although this is a good check of the Monte Carlo algorithm, a formal statistical test (Z-test) test was applied to check whether the solution obtained from the deterministic KCE and the averages over 1000 realizations of the Monte 


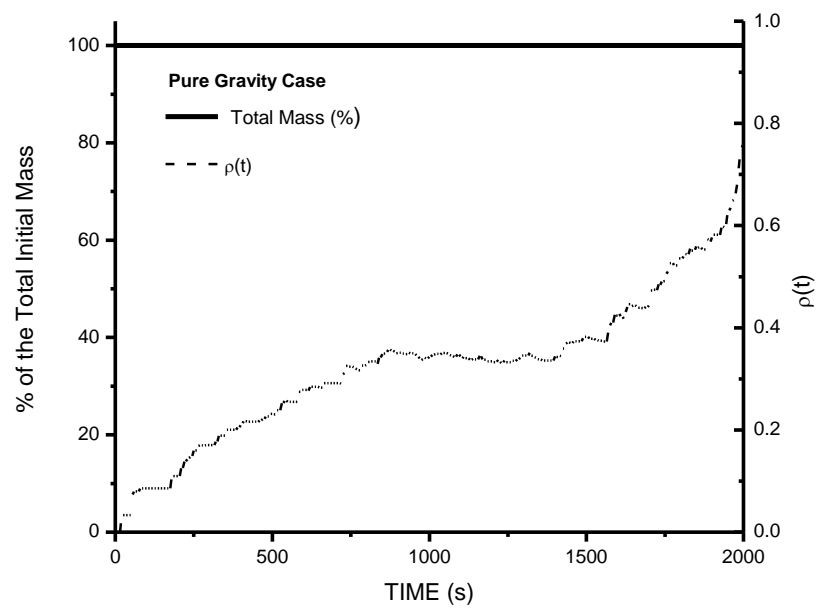

Fig. 3. Same as Fig. 2 but for the pure gravity case.

Carlo method are equal. The null hypothesis would be $\mathrm{H}_{0}$ : $\langle N\rangle=N_{\mathrm{KCE}}$, where $N_{\mathrm{KCE}}$ is the sol concentration calculated from the KCE, and $\langle N\rangle$ is the true stochastic average calculated using the Monte Carlo method (Eq. 11)

$$
\langle N\rangle=\frac{1}{K} \sum_{i=1}^{K} N^{i}
$$

In Eq. (11), $N^{i}$ is the sol concentration for each Monte Carlo realization. As expected, at a $5 \%$ significance level, the null hypothesis $\mathrm{H}_{0}:\langle N\rangle=N_{\mathrm{KCE}}$ is rejected only after approximately 900 s due to the breakdown of the deterministic KCE as the sol-gel transition approaches.

Following Gillespie (1975b), the errors of the Monte Carlo algorithm were calculated in order to check the accuracy in the calculation of Eq. (11). They were estimated as the rms (root mean square) of the fluctuations that may be expected to occur about the average (Eq. 11). Then, for the rms fluctuations we have

$$
\begin{aligned}
\sigma(N(t)) & =\left\{\frac{1}{K} \sum_{i=1}^{K}\left[N^{i}(t)\right]^{2}-\left[\frac{1}{K} \sum_{i=1}^{K} N^{i}(t)\right]^{2}\right\} \\
& =\left\langle N^{2}\right\rangle-\langle N\rangle^{2},
\end{aligned}
$$

where $\langle N\rangle$ is the ensemble average calculated according to Eq. (11). The fluctuations about the average are displayed in Fig. 5 as vertical bars together with the time evolution of the sol concentration obtained from the Monte Carlo simulations. If the condition

$\sigma(N(t)) /\langle N(t)\rangle \ll 1$

is fulfilled, then the results found in separate realizations will be practically identical (Gillespie, 1975b) and the estimate $\langle N\rangle$ will be sufficiently accurate. The ratio (13) was calculated in the time interval $[0,1000]$ and the condition was always fulfilled (See Fig. 5), corroborating the accuracy of the calculations of $\langle N\rangle$ for the turbulent collection kernel.

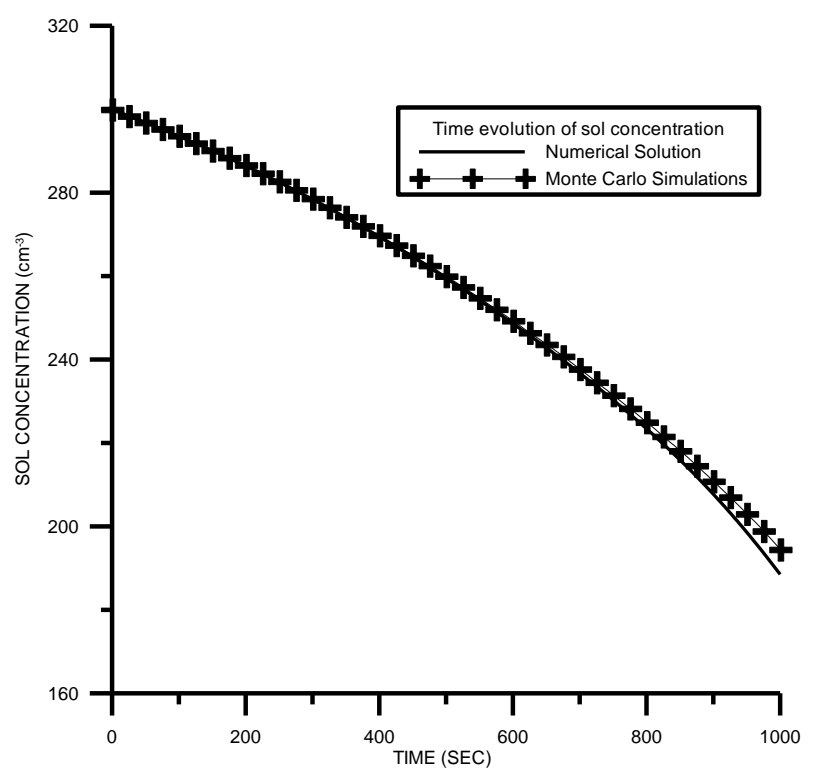

Fig. 4. Time evolution of the sol concentration obtained from the numerical solution of the kinetic collection equation (solid line) and from the Monte Carlo algorithm (crosses).

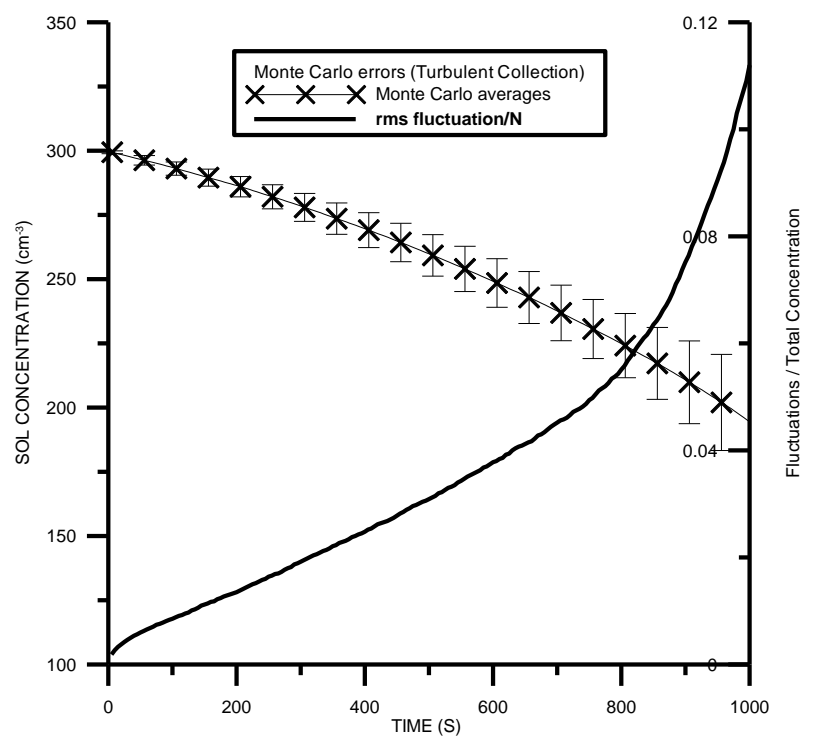

Fig. 5. Time evolution of the sol concentration obtained from Monte Carlo algorithm (crosses and left axis) fluctuations about the average (vertical bars and left axis) and the ratio $\sigma(N(t)) /\langle N(t)\rangle$ (solid line and right axis).

\section{Discussion and conclusions}

One of the outstanding problems in cloud physics is to explain how raindrops can grow by condensation and collisioncoalescence in times as short as $20 \mathrm{~min}$. In order to form a raindrop with a radius of $1 \mathrm{~mm}$ in a warm cloud, a total of $10^{5}$ droplets with radius of $10 \mu \mathrm{m}$ must collide and coalesce. 


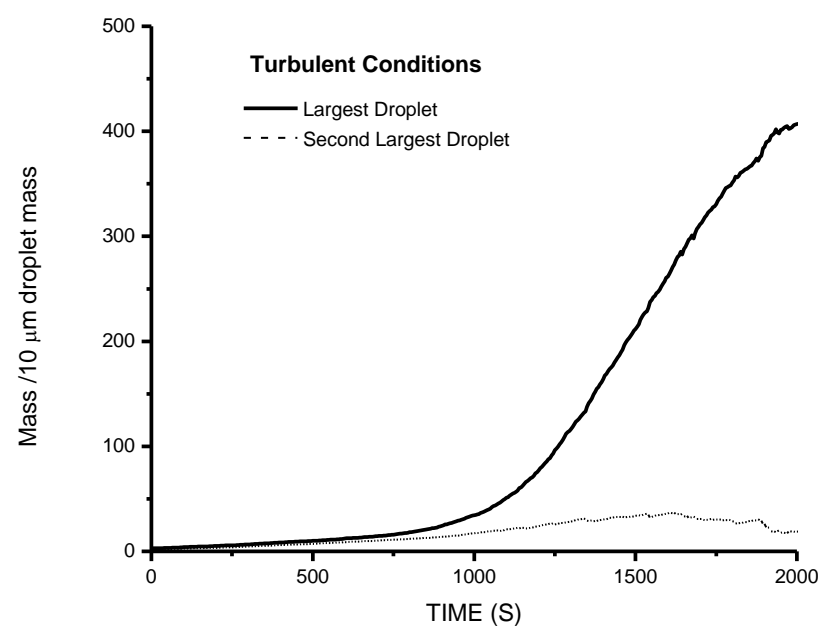

Fig. 6. Time evolution of the ensemble means over all the realizations for the largest (solid line) and second largest (dashed line) droplet masses (expressed in multiples of a $10 \mu \mathrm{m}$ droplet mass) for the turbulent case.

When droplets are small and of uniform size, collisions between them are not very efficient and collision events do not occur with the required rate to produce raindrops until some of the droplets grow to a radius of about $20 \mu \mathrm{m}$.

The fully stochastic turbulent process presented in this study generates a "lucky droplet" that grows faster than the rest of the droplet population. The appearance of a runaway droplet after gelation is a possible mechanism to explain the formation of raindrops. To further clarify this point, we calculate the time evolution of the mass of the largest droplet and second largest droplets as ensemble averages over all the realizations of the fully stochastic simulations. Figure 6 shows the results for the turbulent case, clearly indicating a significant gap between the mass of the largest and second largest droplets after $1000 \mathrm{~s}$. In contrast, the difference in mass in the non-turbulent, pure gravity case shown in Fig. 7 remains much smaller and with no runaway behavior.

The gelation time (Eq. 5) estimated from a set of random realizations constitutes an expected value for the time of the formation of the runaway droplet and has to be compared with the deterministic result obtained from the KCE for a particular system. However, the gelation time is a stochastic variable whose empirical distribution can be calculated from the different realizations of the Monte Carlo algorithm. The problem here is to find a criterion to assess whether a system (for each individual realization) is in runaway growth. For example, Malyshkin and Goldman (2001) declare runaway growth when the coalescence rate of the largest particle is $50 \%$ of the total coalescence rate. Aldous (1997) estimated the runaway time from Monte Carlo simulations via the maximum (over time) of the size of the second largest droplet. A very suitable indicator (Ormel et al., 2010) is $M_{1} / M_{2}$, i.e. the ratio between the mass of the largest to the second largest

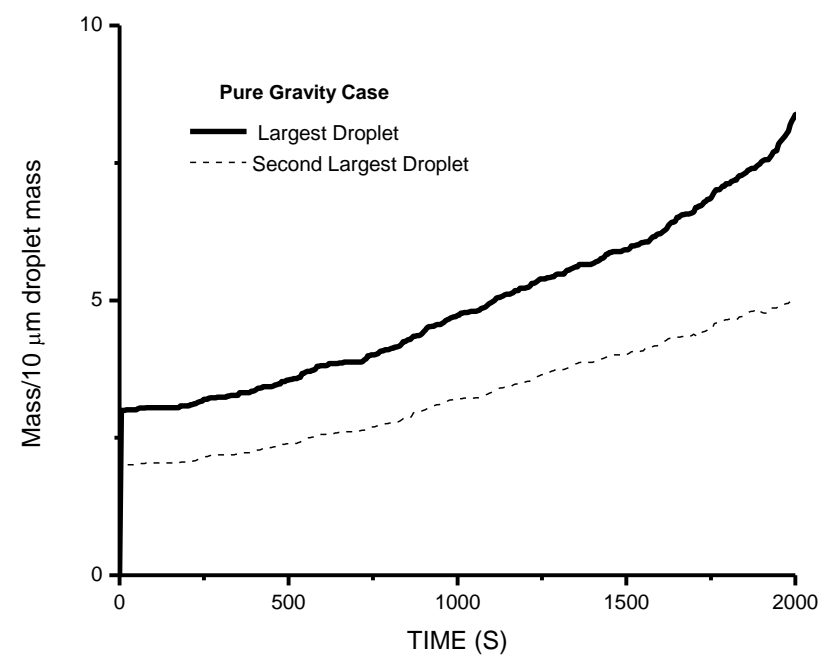

Fig. 7. Same as Fig. 4 but for the pure gravity case.

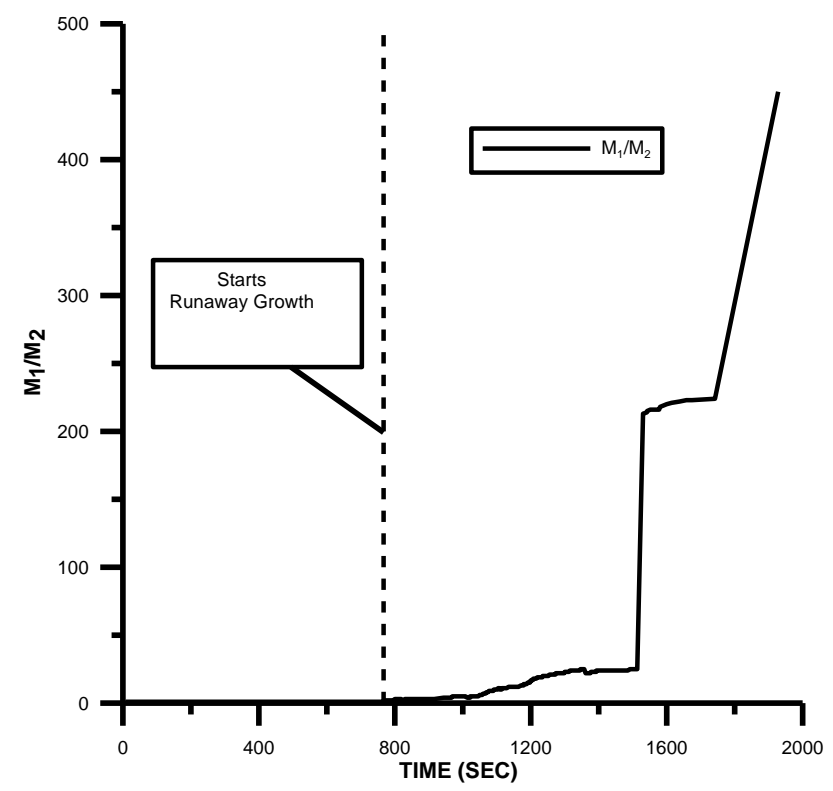

Fig. 8. Time evolution of $M_{1} / M_{2}$ (ratio between the largest and the second largest droplet) for one of the realizations of the stochastic process for the turbulent case.

droplet in the system. When this ratio increases, the system is in runaway growth, otherwise it is not. We have estimated the time evolution of the ratio $M_{1} / M_{2}$ from each realization to determine the time when this ratio started to increase (Fig. 8 shows the time evolution of the ratio $M_{1} / M_{2}$ for one of the realizations). Thus, an empirical distribution for the gelation times was obtained by generating 1000 realizations of the Monte Carlo algorithm (Fig. 9). A similar approach was taken to generate the distribution for the runaway particles radii at the gelation time (Fig. 10). 


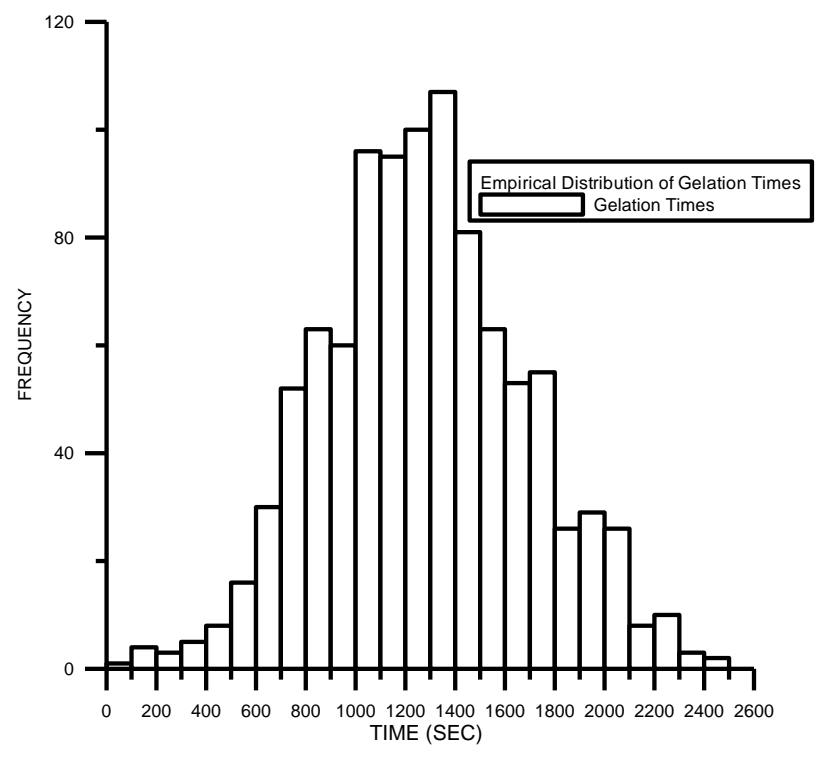

Fig. 9. Empirical distribution for the gelation times obtained for the turbulent case. The results were generated from 1000 realizations of the Monte Carlo algorithm.

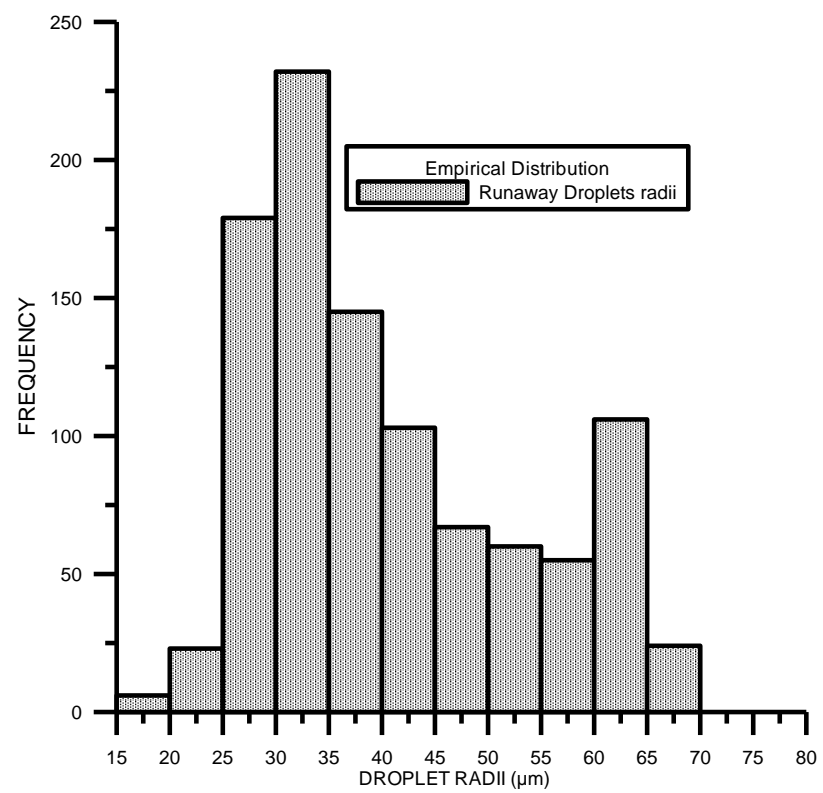

Fig. 10. Empirical distribution for the radius of the runaway droplets. The results were generated from 1000 realizations of the Monte Carlo algorithm.

Kostinski and Shaw (2005) present a distribution of the time to produce drizzle by calculating the convolution of the exponentially distributed times between collisions. They found the distribution of $T_{N_{\mathrm{c}}}=\sum_{i=1}^{N_{\mathrm{c}}} t_{i}$, with $N_{\mathrm{c}}$ fixed, where the $t_{i}$ are the times between droplet collisions and $N_{\mathrm{c}}$ the number of collisions, which have an exponential distribution.
However, in our case the value of $N$ varies, as the number of collisions required to form a runaway droplet is different for each realization of the Monte Carlo process.

The fully stochastic simulations under turbulent conditions performed here include a collision enhancement factor for collisions between droplets with radii $\leq 21 \mu \mathrm{m}$, so the role of turbulence in producing the runaway droplet is likely underestimated in the present study. Since the nucleation and condensation processes are not yet included in this model, future developments will attempt to include the combined effect of turbulent collection and condensation (McGraw and Liu, 2003) on droplet growth.

\section{Appendix A}

\section{The Monte Carlo algorithm}

The stochastic algorithm developed by Gillespie (1976) for chemical reactions was reformulated to simulate the kinetic behavior of aggregating systems by Laurenzi et al. (2002) by defining species as a type of aggregate with a specific size and composition. We have applied this formulation to droplets of different sizes under turbulent conditions.

Within this framework, there is a unique index $\mu$ for each pair of droplets $i, j$ that may collide. For a system with $N$ species $\left(S_{1}, S_{2}, \ldots, S_{N}\right), \mu \in \frac{N(N+1)}{2}$. The set $\{\mu\}$ defines the total collision space, and is equal to the total number of possible interactions. With this set the collision probability density function $P(\tau, \mu)$ can be determined. This quantity is defined by

$P(\tau, \mu) \mathrm{d} \tau \equiv$ Probability that at time $t$ the next collisionin

volume $V$ will occur in the infinitesimal interval

$(t+\tau, t+\tau+\mathrm{d} \tau)$ and will be a $\mu$ collision.

Gillespie (1976) derived this probability density function for a system of $N$ species as

$P(\tau, \mu) \mathrm{d} \tau=a_{\mu} \exp \left(-\sum_{j=1}^{\frac{N(N+1)}{2}} a_{j} \tau.\right)$

Here $\mu \in \frac{N(N+1)}{2}$. The functions $a_{\mu}$ are calculated according to

$a(i, j)=V^{-1} K(i, j) n_{i} n_{j} \mathrm{~d} t \equiv \operatorname{Pr}\{$ Probability that two

unlike particles $i$ and $j$ with populations (number of particles)

$n_{i}$ and $n_{j}$ will collide within the imminent time interval $\}$ 
$a(i, i)=V^{-1} K(i, i) \frac{n_{i}\left(n_{i}-1\right)}{2} \mathrm{~d} t \equiv \operatorname{Pr}\{$ Probability that two particles of the same species $i$ with population (number of particles) $n_{i}$ collide within the imminent time interval $\}$ (A3) The collision probability density function is the basis of the Monte Carlo algorithm. To calculate the evolution of the system, two random numbers $\tau$ and $\mu$ must be generated. Equation (A1) leads directly to the answers of the aforementioned questions. First, we estimate the probability distribution as a function of time by summing $P(\tau, \mu) \mathrm{d} \tau$ (Eq. A1) over all possible collisions $(\mu)$, which results in

$$
\begin{aligned}
& \begin{aligned}
P_{1}(\tau) \mathrm{d} \tau & =\sum_{\mu=1}^{\frac{N(N+1)}{2}} P(\tau, \mu)=\sum_{\mu=1}^{\frac{N(N+1)}{2}} a_{\mu} \exp \left(-\sum_{\nu=1}^{\frac{N(N+1)}{2}} a_{\nu} \tau\right) \\
& =\alpha \exp (-\alpha \tau) \mathrm{d} \tau
\end{aligned} \\
& \text { with } \alpha=\sum_{\nu=1}^{\frac{N(N+1)}{2}} a_{\nu} .
\end{aligned}
$$

The probability function for reactions can be obtained in a similar way, by integrating the probability density function (pdf) $P(\tau, \mu) \mathrm{d} \tau$ over all $\tau$ from 0 to $\infty$, which results in

$$
P_{2}(\mu)=\frac{a_{\mu}}{\alpha} \text {. }
$$

Equation (A4) shows that the probability of a collision in time follows an exponential distribution. In order to obtain a random pair $(\tau, \mu)$, according to the probability density function $P(\tau, \mu)$ we first generate a random number $r_{1}$ distributed uniformly in the interval $(0,1)$; then the inversion method to obtain random numbers is applied. In the inversion method this random number is taken as the probability of a collision in the time period $\tau$ according to $P_{1}(\tau)$. This probability is obtained by integrating $P_{1}(\tau)$ from 0 to $\tau$ :

$r_{1}=\int_{0}^{\tau} P_{1}(z) \mathrm{d} x=\int_{0}^{\tau} \alpha \exp (-\alpha z) d z=1-\exp (-\alpha \tau)(\mathrm{A} 6)$

Considering that $1-r_{1}=r_{1}^{*}$ is also a uniformly distributed random number in the interval $[0,1]$, then the time $\tau$ can be calculated from Eq. (9) in the form

$\tau=\frac{1}{\alpha} \ln \left(\frac{1}{r_{1}^{*}}\right)$.

The collision number $\mu$ is calculated similarly. A random number $r_{2}$ uniformly distributed in the interval $(0,1)$ is generated. Then the pdf $P_{2}(v)$ Eq. (A5) must be integrated over $v$ until the sum of the $\mu$ probability exceeds the random number $r_{2}$. The inequality to obtain the collision index $\mu$ has the form (Gillespie, 1976)

$\sum_{\nu=1}^{\mu-1} a_{\nu}<r_{2} \alpha \leq \sum_{\nu=1}^{\mu} a_{\nu}$

The former results lead to Gillespie's direct algorithm:
1. Initialize (set initial numbers of species, set $t=0$, set stopping criteria).

2. Calculate the function $a_{\mu}$ for all $\mu$.

3. Choose $\tau$ according to the exponential distribution $P_{1}(\tau)=\alpha \exp (-\alpha \tau) \mathrm{d} \tau$.

4. Calculate $\mu$ according to the distribution $P_{2}(\mu)=\frac{a_{\mu}}{\alpha}$.

5. Change the numbers of species to reflect the event of a collision.

6. If stopping criteria are not met, return to step 2 .

Acknowledgements. This study was funded by grants SEPCONACYT 62071 and SEP-CONACYT 131879 from the Consejo Nacional de Ciencia y Tecnología de Mexico, PAPITT-IN105811 and ICyTDF/211/2010.

Edited by: T. Garrett

\section{References}

Aldous, D.: Emergence of the giant component in special MarcusLushnikov process, http://www.stat.berkeley.edu/users/aldous, 1997.

Alfonso, L., Raga, G. B., and Baumgardner, D.: The validity of the kinetic collection equation revisited, Atmos. Chem. Phys., 8, 969-982, doi:10.5194/acp-8-969-2008, 2008.

Alfonso, L., Raga, G. B., and Baumgardner, D.: The validity of the kinetic collection equation revisited - Part 2: Simulations for the hydrodynamic kernel, Atmos. Chem. Phys., 10, 7189-7195, doi:10.5194/acp-10-7189-2010, 2010.

Bayewitz, M. H., Yerushalmi, J., Katz, S., and Shinnar, R.: The extent of correlations in a stochastic coalescence process, J. Atmos. Sci., 31, 1604-1614, 1974.

Drake, R. L. and Wright, T. J.: The Scalar Transport Equation of Coalescence Theory: New Families of Exact Solutions, J. Atmos. Sci., 29, 548-556, 1972.

Gillespie, D. T.: Three models for the coalescence growth of cloud drops, J. Atmos. Sci., 32, 600-607, 1975a.

Gillespie, D. T.: An exact method for numerically simulating the stochastic coalescence process in a cloud, J. Atmos. Sci., 32, 1977-1989, 1975b.

Gillespie, D. T.: A general method for numerically simulating the stochastic time evolution of coupled chemical reactions, J. Comput. Phys., 22, 403-434, 1976.

Hall, W. D.: A detailed microphysical model within a twodimensional dynamic framework: Model description and preliminary results, J. Atmos. Sci., 37, 2486-2507, 1980.

Inaba, S., Tanaka, H., Ohtsuki, K., and Nakazawa, K.: Highaccuracy statistical simulation of planetary accretion: I. Test of the accuracy by comparison with the solution to the stochastic coagulation equation, Earth Planet. Space, 51, 205-217, 1999.

Khain, A., Ovtchinnikov, M., Pinsky, M., Pokrovsky, A., and Krugliak, H.: Notes on the state-of-the-art numerical modeling of cloud physics, Atmos. Res. 55, 159-224, 2000. 
Kostinski, A. B. and Shaw, R. A.: Fluctuations and luck in droplet growth by coalescence, B. Am. Meteor. Soc., 86, 235-244, 2005.

Laurenzi, I. J., Bartels, S. L., and Diamond, S. L.: A general algorithm for exact simulation of multicomponent aggregation, J. Comput. Phys., 177, 418-449, 2002.

Malyshkin, L. and Goodman, J.: The timescale of runaway stochastic coagulation, Icarus, 50, 314-322, 2001.

McGraw, R. and Liu, Y.: Kinetic potential and barrier crossing: a model for warm cloud drizzle formation, Phys. Rev. Lett., 90, 018501, doi:10.1103/PhysRevLett.90.018501, 2003.

Ormel, C. W., Dullemond, C. P., and Spaans, M.: Accretion among planetary bodies: the many faces of runaway growth, Icarus, 210 , 507-538, doi:10.1016/j.icarus.2010.06.011, 2010.

Pinsky, M., Khain, A., and Shapiro, M.: Collisions of small drops in a turbulent flow. Part I: Collision efficiency. Problem formulation and preliminary results, J. Atmos. Sci., 56, 2585-2600, 1999.

Pinsky, M., Khain, A., and Shapiro, M.: Stochastic effects of cloud droplet hydrodynamic interaction in a turbulent flow, Atmos. Res., 53, 131-169, 2000.

Pinsky, M., Khain, A., and Krugliak, H.: Collisions of cloud droplets in a turbulent flow. Part V: Application of detailed tables of turbulent collision rate enhancement to simulation of droplet spectra evolution, J. Atmos. Sci., 65, 357-374, 2008.
Pruppacher, H. R. and Klett, J. D.: Microphysics of clouds and precipitation, Kluwer Academic Publishers, 1997.

Robertson, D.: Monte Carlo simulations of drop growth by accretion, J. Atmos. Sci., 31, 1344-1350, 1974.

Tanaka, H. and Nakazawa, K.: Stochastic coagulation equation and the validity of the statistical coagulation equation, J. Geomag. Geoelecr., 45, 361-381, 1993.

Telford, J. W.: A new aspect of coalescence theory, J. Atmos. Sci., 12, 436-444, 1955.

Vohl, O., Mitra, S. K., Wurzler, S. C., and Pruppacher, H. R.: A wind tunnel study of the effects of turbulence on the growth of cloud drops by collision and coalescence, J. Atmos. Sci., 56, 40884099, 1999.

Wang, L. P., Xue, Y., Ayala, O., and Grabowski, W.: Effects of stochastic coalescence and air turbulence on the size distribution of cloud droplets, Atmos. Res., 82, 416-432, 2006.

Wetherill, G. W.: Comparison of analytical and physical modeling of planetesimal accumulation, Icarus, 88, 336-354, 1990.

Young, K. C.: The evolution of drop spectra due to condensation, coalescence and breakup, J. Atmos. Sci., 32, 965-973, 1975. 